\title{
ORBITS OF PATHS UNDER HYPERBOLIC TORAL AUTOMORPHISMS
}

\author{
RICARDO MAÑÉ
}

\begin{abstract}
A hyperbolic toral automorphism is a map $f: T^{n} \hookleftarrow$ such that has a linear lifting $L: \mathbf{R}^{n} \hookleftarrow$ without eigenvalues of modulus 1 . In this note we prove that the orbit under $f$ of a rectifiable nonconstant path $\gamma:[a, b] \rightarrow T^{n}$ contains a coset of a toral subgroup invariant under same power of $f$. For $C^{2}$ paths the same result was proved by J. Franks. For $C^{0}$ arcs S.G. Hancock proved that it is false.
\end{abstract}

A hyperbolic toral automorphism of $T^{n}=\mathbf{R}^{n} / \mathbf{Z}^{n}$ is a map that lifts to a linear map $L: \mathbf{R}^{n} \hookleftarrow$ without eigenvalues of modulus 1 . Several authors ([1] $[6])$ studied the invariant sets of these transformations. In [2] Franks proved that the closure of the orbit of a nonconstant $C^{2}$ path under a hyperbolic toral automorphism $f$ contains a coset of a toral subgroup invariant under some power of $f$. S. G. Hancock in [3] showed that this property does not extend to $C^{0}$ paths. In this note we shall present a simple proof of an extension of Franks' result to rectifiable paths, i.e. continuous maps $\alpha:[a, b] \rightarrow T^{n}$ such that there exists $K>0$ satisfying $\sum_{n=0} d\left(\alpha\left(t_{n+1}\right)\right.$, $\left.\alpha\left(t_{n}\right)\right) \leqslant K$ for all partition $a=t_{0} \leqslant t_{1} \leqslant \cdots \leqslant t_{n+1}=b$, where $d(\cdot, \cdot)$ denotes any Riemannian metric on $T^{n}$.

THEOREM. Let $\alpha:(a, b) \rightarrow T^{n}$ be a rectifiable nonconstant path and $f: T^{n} \hookleftarrow$ a hyperbolic toral automorphism. Then the closure of the orbit of $\alpha((a, b))$ under $f$ contains a coset of a toral subgroup invariant under some power of $f$.

REMARK I. The toral subgroup cannot be required to be invariant under $f$. For instance if $f_{0}: T^{2} \hookleftarrow$ is a hyperbolic toral automorphism define $f$ : $T^{2} \times T^{2} \hookleftarrow$ as $f(x, y)=\left(y, f_{0}(x)\right)$. This map is a hyperbolic automorphism without proper invariant toral subgroup. However the orbit of any path contained in $T^{2} \times\{e\}$ is not dense in $T^{2} \times T^{2}$.

REMARK II. If the hyperbolic toral automorphism $f: T^{n} \hookleftarrow$ is irreducible in the sense that there are no proper toral subgroups invariant under any power of $f$ then our theorem clearly implies that the orbit of any nonconstant rectifiable path $\alpha:[a, b] \rightarrow T^{n}$ is dense in $T^{n}$. It also implies the following stronger statement: $\alpha([a, b])$ contains transitive points (i.e. points with dense orbit). To see this let $J \subset[a, b]$ be the union of all intervals $\left(t_{1}, t_{2}\right) \subset[a, b]$ such that $\alpha(t)=\alpha\left(t_{1}\right)$ for all $t \in\left(t_{1}, t_{2}\right)$. Let $J^{\prime}=[a, b] \backslash J . J^{\prime}$ is compact

Received by the editors February 27, 1978.

AMS (MOS) subject classifications (1970). Primary 58F10, 58F15.

0002-9939/79/0000-0026/\$02.25

() 1979 American Mathematical Society 
and nonempty. Let $\left\{U_{n} \mid n \in \mathbf{Z}\right\}$ be a basis of open neighborhoods and let $S_{n}=\left\{t \in J^{\prime} \mid f^{m}(\alpha(t)) \in U_{n}\right.$ for some $\left.m\right\}$. Then $S_{n}$ is open and it is dense because if $a \leqslant t_{1}<t_{2} \leqslant b$ belong to $J^{\prime}$ then $\alpha /\left[t_{1}, t_{2}\right]$ is nonconstant, hence its orbit is dense and $f^{m}\left(\alpha\left(\left[t_{1}, t_{2}\right]\right)\right) \cap U_{n} \neq 0$ for some $m$. Then every point in $\bigcap\left\{S_{n} \mid n \in \mathbf{Z}\right\}$ is transitive.

REMARK III. It is easy to construct a nonconstant $C^{0}$ path $\alpha:[a, b] \rightarrow T^{n}$ whose orbit under an irreducible hyperbolic toral automorphism $f$ is dense but that does not contain transitive points. Take a $C^{\infty}$ path $\alpha:[0,1] \rightarrow T^{n}$ such that $\alpha(1 / n)=e$ and $\alpha /[1 /(n+1), 1 / n]$ is nonconstant for all $n \geqslant 1$. Then the orbit of $\alpha /[1 /(n+1), 1 / n]$ is dense in $T^{n}$. By Hancock's results take $\alpha_{n}:[1 /(n+1), 1 / n] \rightarrow T^{n} C^{0}$ near to $\alpha /[1 /(n+1), 1 / n]$ such that $\alpha_{n}(1 / n)=e, \alpha_{n}(1 /(n+1))=e$ and the orbit of $\alpha_{n}([1 /(n+1), 1 / n])$ is not dense. If $\alpha_{n}$ is near enough to $\alpha /[1 /(n+1), 1 / n]$ its orbit fills $T^{n}$ up to $1 / n$. Hence the orbit of the path $\bar{\alpha}:[0,1] \rightarrow T^{n}$ defined by $\bar{\alpha}(t)=\alpha_{n}(t)$ if $t \in$ $[1 /(n+1), 1 / n]$ and $\bar{\alpha}(0)=e$ is dense but the path does not contain transitive points.

Proof OF THE THeORem. Let $\pi: \mathbf{R}^{n} \rightarrow \mathbf{R}^{n} / \mathbf{Z}^{n}$ be the canonical covering map and $d(\cdot, \cdot)$ the flat distance on $T^{n}$. As usual define the length $l(\beta)$ of a path $\beta=[a, b] \rightarrow T^{n}$ (continuous or not) as the supremum of the sums $\sum_{n=0}^{m} d\left(\beta\left(t_{n+1}\right), \beta\left(t_{n}\right)\right)$ over all partitions $a=t_{0} \leqslant \cdots \leqslant t_{m+1}=b$. Define $\sigma:[a, b] \rightarrow \mathbf{R}$ by $\sigma(t)=l(\alpha /[a, t])$. This map is continuous (because $\alpha$ is continuous) and $\sigma\left(t_{1}\right) \geqslant \sigma\left(t_{2}\right)$ if $t_{1} \geqslant t_{2}$. Let $\left[a^{\prime}, b^{\prime}\right]=\sigma([a, b])$ and take $\tau$ : $\left[a^{\prime}, b^{\prime}\right] \rightarrow[a, b]$ satisfying $\sigma \tau(t)=t$ for all $t \in[a, b]$. There always exists such a map (observe that we do not require $\tau$ to be continuous). Let $\alpha_{0}:\left[a^{\prime}, b^{\prime}\right] \rightarrow$ $T^{n}$ defined by $\alpha_{0}=\alpha \tau$. Then $l\left(\alpha_{0} /\left[t_{1}, t_{2}\right]\right)=t_{2}-t_{1}$ for all $a^{\prime} \leqslant t_{1}<t_{2} \leqslant b^{\prime}$. In particular if $\varepsilon>0$ and $\left[a_{k}, b_{k}\right], k=1, \ldots, m$, is a family of disjoint intervals contained in $\left[a^{\prime}, b^{\prime}\right]$ such that $\Sigma_{0}^{m}\left(b_{k}-a_{k}\right) \leqslant \varepsilon$ we have

$$
\sum_{0}^{m} d\left(\alpha\left(a_{k}\right), \alpha\left(b_{k}\right)\right) \leqslant \sum_{0}^{m} l\left(\alpha_{0} /\left[a_{k}, b_{k}\right]\right)=\sum_{0}^{m}\left(b_{k}-a_{k}\right) \leqslant \varepsilon .
$$

This proves that $\alpha_{0}$ is an absolutely continuous path. Take $\gamma:\left[a^{\prime}, b^{\prime}\right] \rightarrow \mathbf{R}^{n}$ absolutely continuous satisfying $\pi \gamma=\alpha_{0}$. Then, by well-known properties of absolutely continuous functions, there exists a set $E \subset[a, b]$ with total measure such that $\dot{\gamma}(t)$ exists for all $t \in E$ and:

$$
\int_{t_{1}}^{t_{2}} \dot{\gamma}(s) d s=\gamma\left(t_{2}\right)-\gamma\left(t_{1}\right)
$$

for all $a^{\prime} \leqslant t_{1} \leqslant t_{2} \leqslant b^{\prime}$. Now observe that we can suppose that the eigenvalues of the linear lifting $L: \mathbf{R}^{n} \hookleftarrow$ of $f$ are either real and positive or are not roots of real numbers because if $L$ does not satisfy this property we can replace $f$ by a suitable power. Observe also that to prove the theorem it is sufficient to find an $f$-invariant toral subgroup $M$ such that for all $\varepsilon>0$ there exists a coset $y+M$ such that all its points can be $\varepsilon$-approximated by points in the orbit of $\gamma\left(\left[a^{\prime}, b^{\prime}\right]\right)$. In order to find $M$ we start defining the subspaces 
$E_{\lambda}$ as $\cup_{n>0}(L-\lambda I)^{-n}(0)$ if $\lambda$ is real or

$$
E_{\lambda}=\left(\left(\bigcup_{n>0}(\hat{L}-\lambda I)^{-n}(0)\right) \cup\left(\bigcup_{n>0}(\hat{L}-\bar{\lambda} I)^{-n}(0)\right)\right) \cap \mathbf{R}^{n}
$$

where $\hat{L}: \mathbf{C}^{n} \hookleftarrow$, is the complexification of $L$, if $\lambda$ is complex. Take eigenvalues $\lambda_{1}, \ldots, \lambda_{m}$ of $L$ such that $\dot{\gamma}(t) \in \bigoplus_{i=1}^{m} E_{\lambda_{i}}$ for all $t \in E$ and for all $\lambda_{j}$ there exists $t \in E$ such that $\dot{\gamma}(t)$ has nonzero component on $E_{\lambda_{j}}$. The set $\lambda_{1}, \ldots, \lambda_{n}$ is nonempty because $\gamma$ is nonconstant. Suppose $\left|\lambda_{1}\right| \geqslant\left|\lambda_{j}\right|$ for all $1 \leqslant j \leqslant m$. Take $t_{0} \in E$ such that $v=\dot{\gamma}\left(t_{0}\right)$ has nonzero projection on $E_{\lambda_{1}}$. Define $p(t)=\gamma(t)-\gamma\left(t_{0}\right)-\left(t-t_{0}\right) v$. Then $\lim _{t \rightarrow t_{0}} p(t) /\left(t-t_{0}\right)=0$ and

$$
p(t)=\int_{t_{0}}^{t} \dot{\gamma}(s) d s-\left(t-t_{0}\right) v .
$$

Hence:

$$
p(t) \in \bigoplus_{i=1}^{m} E_{\lambda_{i}}
$$

for all $t \in[a, b]$. Moreover since every eigenvalue of $L$ has modulus $\neq 1$ we can assume $\left|\lambda_{1}\right|>1$ (otherwise replace $f$ by $f^{-1}$ ). Define $\tilde{L}: S^{n-1} \hookleftarrow$ by $\tilde{L} w=L w /\|L w\|$. Using the real canonical form of $L$ and the fact that every eigenvalue of $L$ is either real and positive or has no real powers it is easy to prove that the limit $\Lambda$ set of $v /\|v\|$ under $\tilde{L}$ is a connected, minimal set for $\tilde{L}$ and generates an $L$-invariant subspace $S$ such that $\|L w\|=\left|\lambda_{1}\right|\|w\|$ for all $w \in S$. Let $M$ be the closure of $\pi(S) . M$ is a toral subgroup. Take $w \in \Lambda$ such that $\pi(\{\lambda w \mid \lambda>0\})$ is dense in $M$. Given $\varepsilon>0$ take $T>0$ such that $d_{0}(\pi(\{\lambda w \mid 0<\lambda<T\}), M)<\varepsilon / 3$, where $d_{0}(\cdot, \cdot)$ denotes the Hausdorff metric. Let $\delta>0$ be such that if $\bar{w} \in \mathbf{R}^{n},\|\bar{w}\|=1$ and $\|\bar{w}-w\| \leqslant \delta$ then $d(\pi(\{\lambda \bar{w} \mid 0<\lambda<T\}), M)<\varepsilon / 2$. Since the limit set of $v /\|v\|$ under $\tilde{L}$ is a minimal set for $\tilde{L}$ we can find an increasing sequence $\left\{n_{k} \mid k \geqslant 0\right\}$ of positive integers such that:

$$
\begin{aligned}
& \lim _{k \rightarrow+\infty} n_{k}=\infty, \\
& \left\|L^{n_{k}} v /\right\| L^{n_{k}} v\|-w\|<\delta, \quad k \geqslant 0, \\
& \sup \left\{n_{k+1}-n_{k} \mid k \geqslant 0\right\}<\infty \text {. }
\end{aligned}
$$

From (3) we can define:

$$
K=\sup \left\{\left\|L^{n_{k+1} v}\right\| /\left\|L^{n_{k}} v\right\| \mid k \geqslant 0\right\} .
$$

Moreover there exist constants $C_{1}>0, C>0$ such that:

$$
C_{1}\left\|L^{n} v\right\| \geqslant\left|\lambda_{1}\right|^{n}\|v\|, \quad\left\|L^{n} w\right\| \leqslant C\left|\lambda_{1}\right|^{n}\|w\|
$$

for all $w \in \bigoplus_{i=1}^{m} E_{\lambda_{i}}, n \geqslant 0$. Now choose $\delta_{0}>0$ satisfying

$$
\|p(t)\| \leqslant \frac{\varepsilon}{2 C C_{1} K T}\left|t-t_{0}\right|\|v\|
$$


for all $\left|t-t_{0}\right| \leqslant \delta_{0}$. By (1) and (5) there exists $n_{k}$ such that:

$$
\left\|L^{n_{k}}\left(\delta_{0} v\right)\right\|>T, \quad\left\|L^{n_{k-1}}\left(\delta_{0} v\right)\right\| \leqslant T .
$$

The definition of $K$ implies:

$$
\left\|L^{n_{k}}\left(\delta_{0} v\right)\right\| \leqslant K T
$$

We claim that:

$$
\left\|L^{n_{k}}\left(\gamma(t)-\gamma\left(t_{0}\right)\right)-L^{n_{k}}\left(t-t_{0}\right) v\right\| \leqslant \varepsilon / 2
$$

if $\left|t-t_{0}\right| \leqslant \delta_{0}$. By (0), (4), (5), (6) and (8):

$$
\begin{aligned}
&\left\|L^{n_{k}}\left(\gamma(t)-\gamma\left(t_{0}\right)\right)-L^{n_{k}}\left(t-t_{0}\right) v\right\|=\left\|L^{n_{k}} p(t)\right\| \\
& \leqslant C\left|\lambda_{1}\right|^{n_{k}}\|p(t)\| \leqslant C\left|\lambda_{1}\right|^{n_{k}} \frac{\varepsilon}{2 C C_{1} K T}\left|t-t_{0}\right|\|v\| \\
& \leqslant \frac{C \varepsilon}{2 C C_{1} K T}\left|\lambda_{1}\right|^{n_{k}} \delta_{0}\|v\| \leqslant \frac{C \varepsilon}{2 C C_{1} K T} C_{1}\left\|L^{n_{k}}\left(\delta_{0} v\right)\right\| \\
& \leqslant \frac{C \varepsilon}{2 C C_{1} K T} C_{1} K T=\frac{\varepsilon}{2} .
\end{aligned}
$$

Now consider the coset $f^{n_{k}}\left(\alpha_{0}\left(t_{0}\right)\right)+M$. If $x \in M$ by (2) we can take $0<t<T$ such that

$$
d\left(\pi\left(t \frac{L^{n_{k}} v}{\left\|L^{n_{k} v}\right\|}\right), x\right) \leqslant \varepsilon / 2 .
$$

Write $t L^{n_{k}} v /\left\|L^{n_{k}} v\right\|=L^{n_{k}} t_{1} v$ with $t_{1}=t /\left\|L^{n_{k}} v\right\|$. By (7) $0<t_{1}<\delta_{0}$. Then:

$$
\begin{aligned}
d\left(f^{n_{k}}\left(\alpha_{0}\left(t_{0}+t_{1}\right)\right), f^{n_{k}}\left(\alpha\left(t_{0}\right)\right)+x\right) & \\
\leqslant & \frac{\varepsilon}{2}+d\left(f^{n_{k}}\left(\alpha\left(t_{0}+t_{1}\right)\right), f^{n_{k}}\left(\alpha\left(t_{0}\right)\right)+L^{n_{k}} t_{1} v\right) \\
\leqslant & \frac{\varepsilon}{2}+\left\|L^{n_{k}}\left(\gamma\left(t_{0}+t_{1}\right)-\gamma\left(t_{0}\right)\right)-L^{n_{k}} t_{1} v\right\|
\end{aligned}
$$

and using (9):

$$
d\left(f^{n_{k}}\left(\alpha_{0}\left(t_{0}+t_{1}\right)\right), f^{n_{k}}\left(\alpha\left(t_{0}\right)\right)+x\right) \leqslant \varepsilon / 2+\varepsilon / 2=\varepsilon .
$$

Therefore we found a coset of $M$ such that all its points can be $\varepsilon$-approximated by points in the orbit of $\alpha_{0}\left(\left[a^{\prime}, b^{\prime}\right]\right)$ as we wanted to prove.

REMARK IV. The theorem is also true for nonconstant paths $\alpha:[a, b] \rightarrow T^{n}$ such that $\dot{\alpha}(t)$ exists for all $t \in[a, b]$. Even more general, the theorem is valid for nonconstant paths $\alpha:[a, b] \rightarrow T^{n}$ with the following property: $(\mathscr{P})-\alpha$ has a lifting $\gamma:[a, b] \rightarrow \mathbf{R}^{n}$ such that for every subspace $G$ if there exist $a \leqslant t_{1} \leqslant t_{2} \leqslant b$ with $\gamma\left(t_{1}\right)-\gamma\left(t_{2}\right) \notin G$ then for some $t \in[a, b], \dot{\gamma}(t)$ exists and does not belong to $G$.

In particular, paths $\alpha:[a, b] \rightarrow T^{n}$ with derivative at every point satisfy this property because if $\gamma:[a, b] \rightarrow \mathbf{R}^{n}$ is a lifting of $\alpha, G$ a subspace and $\gamma\left(t_{1}\right)-\gamma\left(t_{2}\right) \notin G$ then taking linear maps $\varphi_{i}: \mathbf{R}^{n} \rightarrow \mathbf{R}, i=1, \ldots, k$, such 
that $\cap_{i=1}^{k} \varphi_{i}^{-1}(0)=G$ either $\dot{\gamma}(t) \notin G$ for some $t$ or $\varphi_{i} \gamma$ is constant for all $i=1, \ldots, k$. Hence $\varphi_{i}\left(\gamma\left(t_{1}\right)-\gamma\left(t_{2}\right)\right)=\varphi_{i} \gamma\left(t_{1}\right)-\varphi_{i} \gamma\left(t_{2}\right)=0$ for all $i=$ $1, \ldots, k$ thus implying $\gamma\left(t_{1}\right)-\gamma\left(t_{2}\right) \in G$.

For paths satisfying $(\mathscr{P})$ the proof of the theorem is the same as previously with minor changes. Define $E \subset[a, b]$ as $E=\{t \in[a, b] \mid \dot{\gamma}(t)$ exists $\}$. Then define $\bigoplus_{i=1}^{m} E_{\lambda_{i}}$, choose $t_{0}$ and define $p(t)$ as we did before (replacing $\gamma$ : $\left[a^{\prime}, b^{\prime}\right] \rightarrow \mathbf{R}^{n}$ by $\left.\gamma:[a, b] \rightarrow \mathbf{R}^{n}\right)$. To prove the relation $p(t) \in \bigoplus_{i=1}^{m} E_{\lambda_{i}}$ for all $t \in[a, b]$ observe that if $p(t) \notin \bigoplus_{i=1}^{m} E_{\lambda_{i}}$ for some $t \in[a, b]$ then $\gamma(t)-\gamma\left(t_{0}\right)$ $=\left(t-t_{0}\right) v+p(t) \notin \bigoplus_{i=1}^{m} E_{\lambda_{i}}$. Hence for some $t_{1} \in[a, b], \dot{\gamma}\left(t_{1}\right)$ exists and does not belong to the subspace $\bigoplus_{i=1}^{m} E_{\lambda_{i}}$ thus contradicting its definition. After this modification the proof remains the same.

\section{REFERENCES}

1. R. Bowen, Markov partitions are not smooth, Proc. Amer. Math. Soc. 71 (1978), 130-132.

2. J. Franks, Invariant sets of hyperbolic toral automorphisms, Amer. J. Math. 99 (1977), 1089-1095.

3. S. G. Hancock, Orbits of paths under hyperbolic toral automorphisms, Bull. London Math. Soc. (to appear)

4. M. Hirsch, On invariant subsets of hyperbolic sets, Essays in Topology and Related Topics, Springer-Verlag, Berlin, 1970.

5. R. Mañé, Invariant sets of Anosov diffeomorphisms, Invent. Math. 46 (1978), 147-152.

6. F. Przytcki, Construction of invariant sets for Anosov diffeomorphisms and hyperbolic attractors, Polish Academy of Sciences, preprint 119.

Instituto de Matematica Pura e Aplicada, Rua luiz de Camoes, 68, 20.000 Rio de JANEIRO, R. J., BRAZIL 